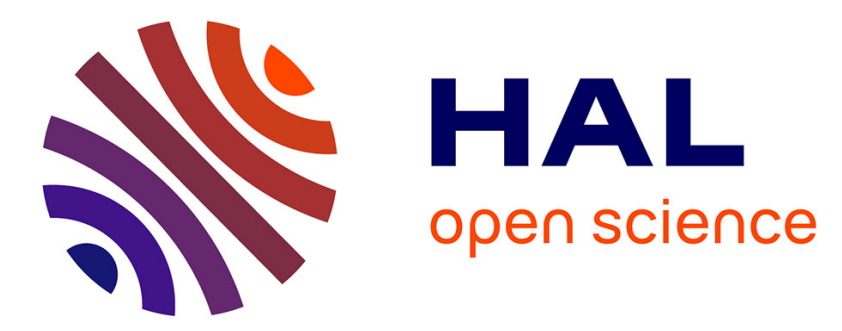

\title{
Contact-Less Measurement System for Cardiopulmonary Activity
}

Sarah El-Samad, Dany Obeid, Sawsan Sadek, Gheorghe Zaharia, Ghaïs El Zein

\section{- To cite this version:}

Sarah El-Samad, Dany Obeid, Sawsan Sadek, Gheorghe Zaharia, Ghaïs El Zein. Contact-Less Measurement System for Cardiopulmonary Activity. 14th Mediterranean Microwave Symposium, Dec 2014, Marrakech, Morocco. hal-01100638

\section{HAL Id: hal-01100638 \\ https://hal.science/hal-01100638}

Submitted on 6 Jan 2015

HAL is a multi-disciplinary open access archive for the deposit and dissemination of scientific research documents, whether they are published or not. The documents may come from teaching and research institutions in France or abroad, or from public or private research centers.
L'archive ouverte pluridisciplinaire HAL, est destinée au dépôt et à la diffusion de documents scientifiques de niveau recherche, publiés ou non, émanant des établissements d'enseignement et de recherche français ou étrangers, des laboratoires publics ou privés. 


\title{
Contact-Less Measurement System for Cardiopulmonary Activity
}

\author{
Sarah El-Samad ${ }^{1,2}$, Dany Obeid ${ }^{1}$, Sawsan Sadek ${ }^{1}$, Gheorghe Zaharia ${ }^{2}$, Ghais El Zein ${ }^{2}$ \\ ${ }^{1}$ Doctoral School of Science and Technology, Lebanon \\ ${ }^{2}$ IETR - UMR CNRS 6164, INSA Rennes, France \\ Sarah.samad@insa-rennes.fr
}

\begin{abstract}
This paper presents a wireless cardiopulmonary activity measurement system. This system generates a continuous wave toward a person's chest set at a distance of $1 \mathrm{~m}$, then reflected to the system. Using a vector network analyzer, the phase of $S_{21}$ is computed. The phase variation of $S_{21}$ contains information about cardiopulmonary activity. Several processing techniques are used to separate heartbeat signal from cardiorespiratory signal either in frequency or in temporal domain. The measurements were performed simultaneously with a PC-based electrocardiogram to validate the heartbeat rate detection techniques. In conclusion, processing techniques used in this paper give accurate results.
\end{abstract}

Keywords- heartbeat detection; wireless monitoring; Doppler radar; electrocardiogram; signal processing

\section{INTRODUCTION}

Nowadays, contact-less monitoring patient's heartbeat using Doppler radar has attracted considerable interest of researchers, especially when the traditional electrocardiogram (ECG) measurements with fixed electrodes is not practical in some cases like infants at risk or sudden infant syndrome or burn victims [1], [2]. According to Doppler effect, a constant frequency signal reflected off an object having a varying displacement will result in a reflected signal, but with a time varying phase [3]. In our case, the object is the patient's chest; the reflected signal of the person's chest contains information about the heartbeat and respiration [1], [4]. The average peakto-peak chest motion due to respiration is between 4 and 12 $\mathrm{mm}$, and due to the heartbeat alone is between 0.2 and 0.5 $\mathrm{mm}$. Moreover, at rest, the respiration frequency varies between 0.1 and $0.3 \mathrm{~Hz}$. Hence, the respiration rate varies between 6 and 18 beats per minute (bpm). Moreover, the heartbeat frequency changes within the 1 and $3 \mathrm{~Hz}$ interval. Hence, the heartbeat rate varies between 60 and 180 bpm [1], [4].

Previous works use systems with fixed frequency and power. The first system generates a $9 \mathrm{GHz}$ continuous wave and is based on Doppler effect [5]. The detected signal is displayed on an oscilloscope. Order 4 Chebychev band-pass filter is applied between 0.5 and $1.5 \mathrm{~Hz}$ to finally detect the heartbeat signal. The detection is done in real time [5], [6]. Another system consists of a microwave source, a directional coupler, a pump, a mixer, a filter, a digital signal processing card PCMCIA having a 12-bit A/D converter and two separate pairs of patch antennas for transmit and receive functions [7]. In this reference, authors have also reported results with a single antenna, using a circulator or a directional coupler. The received signal is converted to baseband using a small portion of the signal originated from the local oscillator. The hardware filters for heart pattern can be bulky using digital signal processing. To determine rates, peak detection method is used. Fig. 1 presents the schematic of the system.

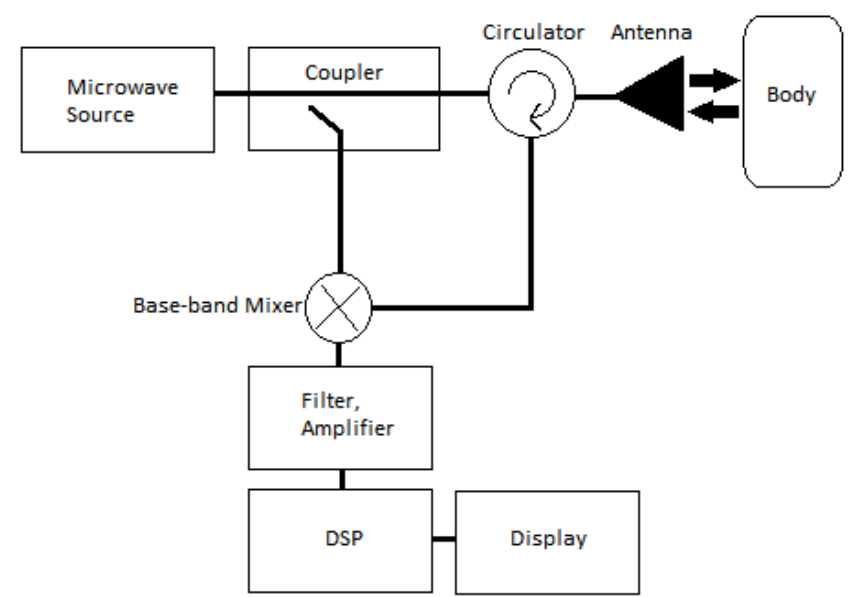

Figure 1. Measurement system with a single antenna [7].

Recently, a system was designed for heartbeat detection with the ability of tuning both frequency and power in order to separate the heart and respiration signals [1], [4], [8]. The simplicity of the system installation is useful in order to determine the optimal frequency with the minimum power before the implementation process; this feature does not exist in the previous systems [1], [5]. The elimination of low frequencies of the received signal is applied especially because breathing amplitude is greater than heartbeat amplitude. To remove the low frequency which represents the breathing frequency, high-pass filter (HPF) or band-pass filter (BPF) could be applied. Several kinds of digital filters can be used in this case, such as finite impulse response filter (FIR) or infinite impulse response filters (IIR). Filter properties are determined by the number and the values of its coefficients and the filter order. The properties of the frequency response of these filters include the cutoff frequency, the steepness of the transition between the pass-band and stop-band, and the 
amount or ripples in the pass-band and stop-band. In addition, wavelet transform was recently applied to extract the heart rate with high performance. Two types of wavelets can be used: 'Discrete Wavelet Transform' and 'Continuous Wavelet Transform'. In our system, 'Discrete Wavelet Transform' (DWT) is used. DWT is computed by successive low-pass and high-pass filtering of the discrete time-domain signal. There are different families of wavelets. This makes different tradeoffs between how compactly the basic functions are localized in space and how smooth they are. Each family of wavelets has subclasses distinguished by the number of coefficients and by the level of iterations [1].

All these methods are applied to mathematical models where heartbeat and respiration signals are mixed. In this work, real measurement data of human body's chest movement are performed. The person is sitting in front of the Doppler radar at one meter. In order to validate the used signal processing method, on-body ECG measurements are also performed using the same start time. Previous work on real measurements were made by cutting the breath, and then compared to the ECG [9]. In the present work, breathing will be considered. The rest of the paper is organized as follows: description of the proposed microwave system is presented in Section II. The heartbeats detection in frequency domain is shown in Section III. Section IV shows the obtained results of the heart beats detection in the time domain. Finally, the conclusion is given in Section V.

\section{SYSTEM DESCRIPTION}

The used system is based on a Vector Network Analyzer (VNA) and two horn antennas. The VNA has several features, and the ability of tuning both frequency and power of the transmitted signal. Moreover, it measures the time variation of the phase of the transmission coefficient $S_{21}$. This phase corresponds to the difference between the phase of the received and the transmitted signal. This installation is useful in order to determine the optimal frequency with the minimum power before implementation process. The VNA generates a Continuous Wave $(\mathrm{CW})$ signal at $5.8 \mathrm{GHz}$. The emitted power is $0 \mathrm{dBm}$ and the antenna gain is $11 \mathrm{~dB}$. The reflected signal that carries chest information is received by the $\mathrm{Rx}$ antenna and fed back into the VNA where the phase of $\mathrm{S}_{21}$ is computed [1]. A 27 years old person is sitting in front of the system at a distance of $1 \mathrm{~m}$. The sampling frequency is $1250 \mathrm{~Hz}$; the measurement is realized in 16 seconds. The person is breathing normally. To validate the proposed system, ECG measurements are started simultaneously with the system measurements and then compared. Fig. 2 presents the contact less system and the contact ECG system. Fig. 3 presents the detected phase variation of $\mathrm{S}_{21}$ signal. Note that after the FFT transform of the signal, we obtain $0.1875 \mathrm{~Hz}$ as respiration frequency, and hence the respiration rate is about $11.25 \mathrm{bpm}$. In this work, heartbeat rate is extracted in time domain and frequency domain in order to separate the respiratory signal from heartbeat signal. To extract heartbeat rate in temporal domain, peak detection is used after applying high-pass filter and smoothing. Moreover, band-pass filter can be applied. In frequency domain, after FFT operator high-pass filter is used. The maximum value between 0.5 and $3 \mathrm{~Hz}$ corresponds to the heartbeat rate frequency.

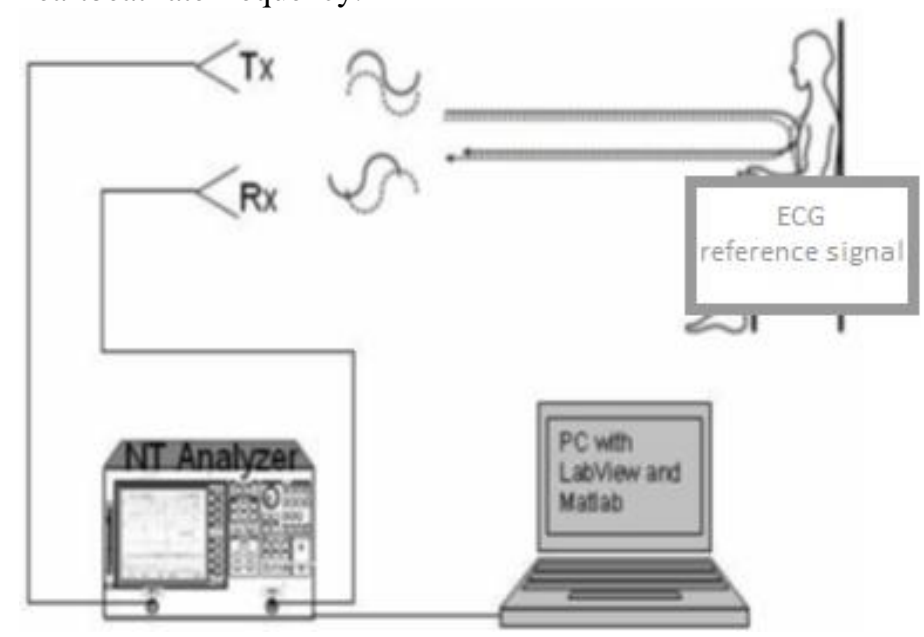

Figure 2. Proposed measurement setup.

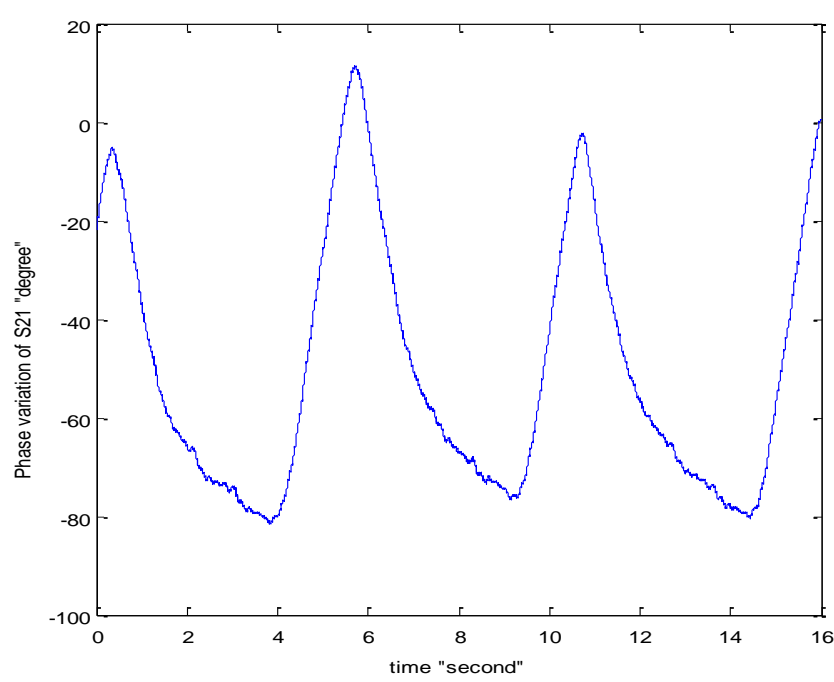

Figure 3. Phase of the $S_{21}$ signal.

\section{HEARTBEAT DETECTION IN FREQUENCY DOMAIN}

Fig. 4 shows the results of Fast Fourier Transform (FFT) applied to the received signal. Note that ' $0.1875 \mathrm{~Hz}$ ', which represents the position of the peak that has the maximum amplitude, is the respiratory frequency. However, the problem of heartbeat's low amplitude exists, and hence the detection of the heartbeat frequency is quite difficult. To detect this frequency, high-pass filter (HPF) is applied with $0.9 \mathrm{~Hz}$ as cut-off frequency. The high-pass filter is a Butterworth filter with order 4. Fig. 5 shows the results of applying Fast Fourier Transform (FFT) to the received signal after the high-pass filter. A value of $0.9 \mathrm{~Hz}$ is chosen as cut-off frequency, because it eliminates respiratory frequencies and provides at its output the heartbeat frequencies. The position of the peak with the maximum amplitude represents the heartbeat 
frequency. The value of detected heartbeat frequency is 1.188 $\mathrm{Hz}$ which corresponds to $71 \mathrm{bpm}$.

In parallel, ECG is used to verify the measurements accuracy. The sampling frequency of the ECG is $360 \mathrm{~Hz}$. Peak detection was applied to the signal. 18 peaks were detected from the ECG signal. The obtained frequency of the heartbeat is $1.19 \mathrm{~Hz}$, corresponding to $71 \mathrm{bpm}$. Fig. 6 presents the peak detection of the ECG signal. The value detected from the contact-less system is close to the heartbeat frequency detected from the ECG. This value gives $99 \%$ accuracy.

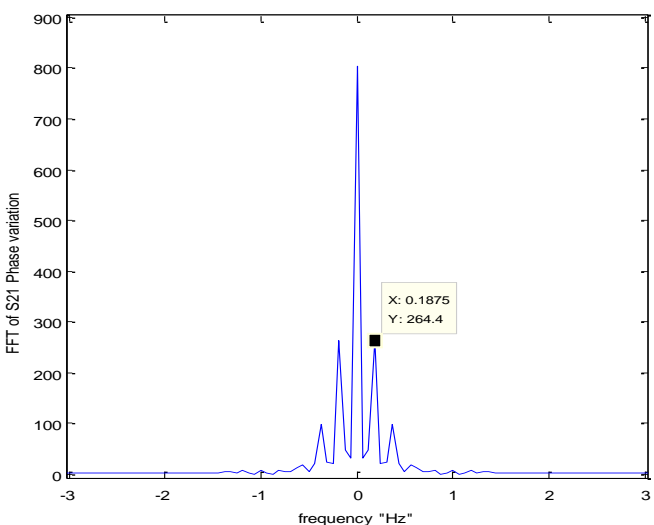

Figure 4. FFT of the phase of $S_{21}$.

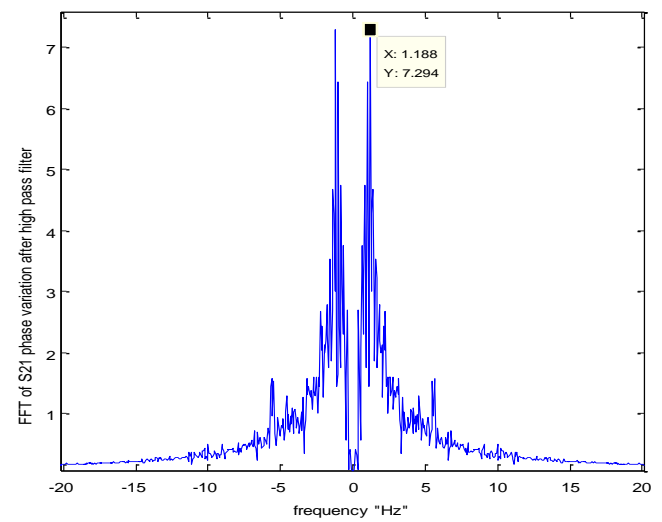

Figure 5. FFT of the phase of $\mathrm{S}_{21}$ after high-pass filter.

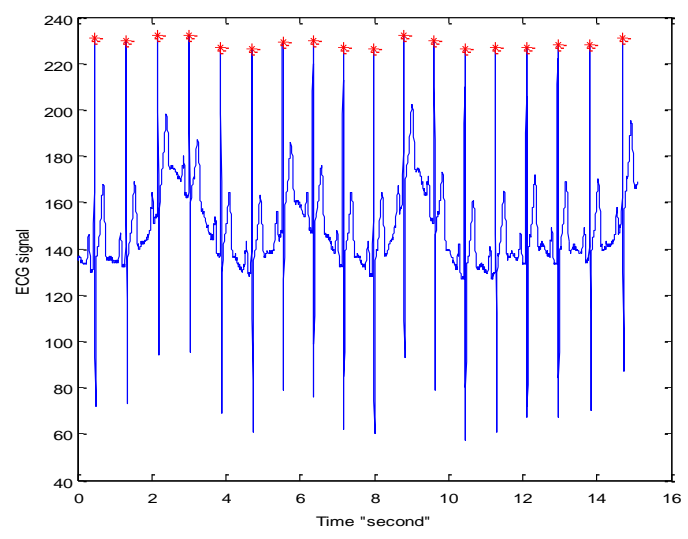

Figure 6. Peak detection of the ECG signal.

\section{HEARTBEAT DETECTION IN TIME DOMAIN}

To detect heartbeat rate in time domain, filtering of the phase of $S_{21}$ is applied, followed by peak detection. Fig. 7 presents the peak detection after applying 4-order Butterworth band-pass filter, having $0.9 \mathrm{~Hz}$ and $2 \mathrm{~Hz}$ as cutoff frequencies: $0.9 \mathrm{~Hz}$ eliminates respiratory frequencies and $2 \mathrm{~Hz}$ eliminates high-frequency noise. Thus, the heartbeat frequencies are obtained at its output. After applying peak detection to the filtered signal, $1.17 \mathrm{~Hz}$ is obtained as heartbeat frequency which represents $70 \mathrm{bpm}$. On the other side, smoothing method is needed when applying high-pass filter to the phase of $S_{21}$

Therefore, the high-pass filtered signal is smoothed by a MATLAB function. This function uses sliding average of length $N$. Sliding average takes the average of every $N$ consecutive samples of the waveform. After trial, the length of the window ' $\mathrm{N}$ ' is chosen to be 200 as an optimal value for higher accuracy detection, then peak detection is applied. Figs. 8 and 9 present the peak detection before and after smoothing method applied to the high-pass filtered phase of $S_{21}$. A 4 order Butterworth filter is used, having $0.8 \mathrm{~Hz}$ as cutoff frequency.

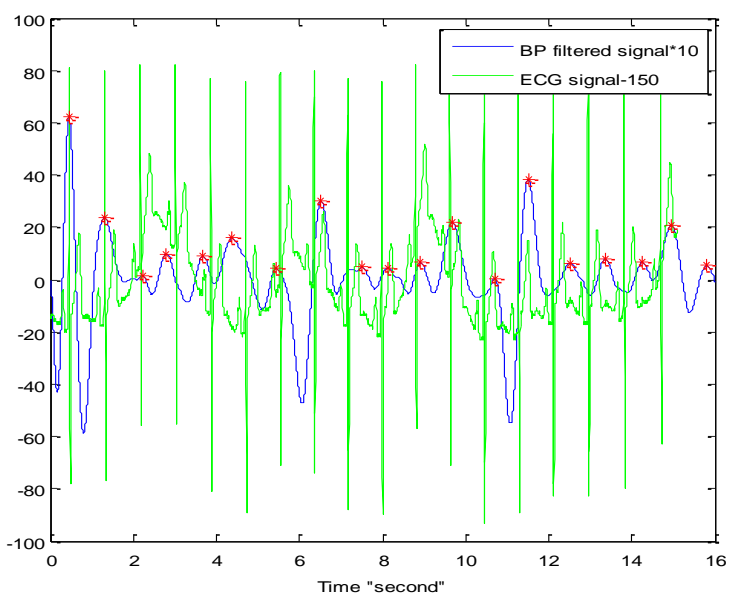

Figure 7. Peak detection after band-pass filtered phase of $S_{21}$.

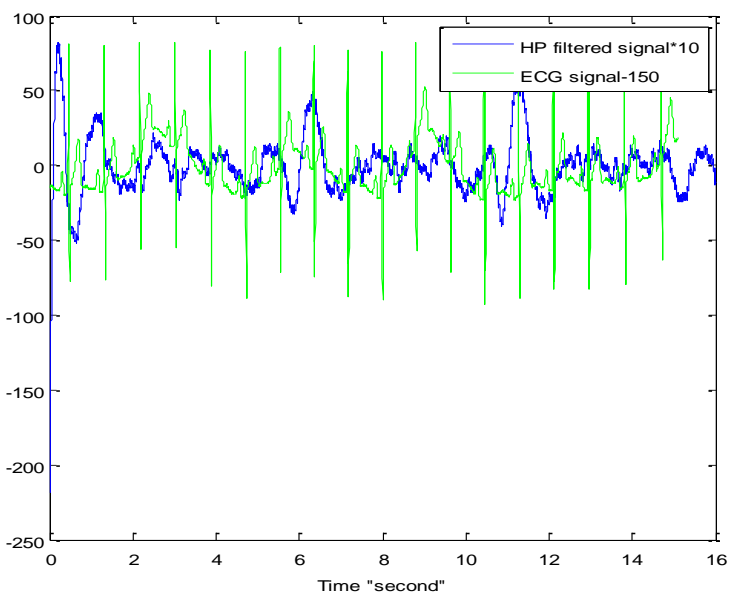

Figure 8. Phase of $S_{21}$ after applying high-pass filter. 


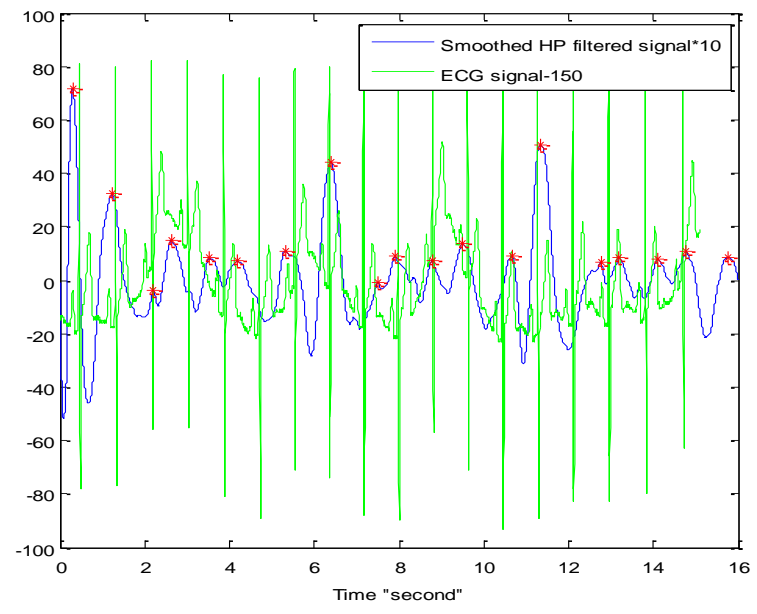

Figure 9. Peak detection of the phase of $S_{21}$ after applying high-pass filter and smoothing.

Several filters with different parameters are applied to get the heart-beat rate. These results are compared with the similar ones obtained from the ECG signal used as reference. ECG gives $1.19 \mathrm{~Hz}$ as heartbeat frequency and $71 \mathrm{bpm}$. Heartbeat frequencies 'HBF', heartbeat rates 'HBR' and accuracies 'Acc' of filters are summarized in Table 1.

\begin{tabular}{|c|c|c|c|c|c|c|}
\hline $\begin{array}{c}\text { Filter } \\
\text { type }\end{array}$ & $\begin{array}{c}\text { Butter } \\
\text { BPF } \\
\text { N=4; } \\
\text { Fc1=0.8 } \\
\text { Hz; } \\
\text { Fc2=2 } \\
\text { Hz. }\end{array}$ & $\begin{array}{c}\text { Butter } \\
\text { BPF } \\
\text { N=4; } \\
\text { Fc1=0.9 } \\
\text { Hz; } \\
\text { Fc2=2 } \\
\text { Hz. }\end{array}$ & $\begin{array}{c}\text { Butter } \\
\text { HPF } \\
\mathrm{N}=4 \text {; } \\
\text { Fc=0.9 } \\
\text { Hz. } \\
\text { with } \\
\text { smoothing }\end{array}$ & $\begin{array}{c}\text { Butter } \\
\text { HPF } \\
\text { N= 4; } \\
\text { Fc = 0.8 } \\
\text { Hz. } \\
\text { with } \\
\text { smoothing }\end{array}$ & $\begin{array}{c}\text { Butter } \\
\text { HPF } \\
\mathrm{N}=4 ; \\
\text { Fc }=0.9 \\
\text { Hz. } \\
\text { without } \\
\text { smoothing }\end{array}$ & $\begin{array}{c}\text { Butter } \\
\text { HPF } \\
\mathrm{N}=4 ; \\
\mathrm{Fc}=0.8 \\
\text { Hz. } \\
\text { without } \\
\text { smoothing }\end{array}$ \\
\hline $\begin{array}{c}\text { HBF } \\
\mathbf{H z}\end{array}$ & 1.17 & 1.168 & 1.169 & 1.175 & 1.172 & 1.036 \\
\hline $\begin{array}{l}\text { HBR } \\
\text { bpm }\end{array}$ & 70 & 70 & 70 & 70 & 70 & 62 \\
\hline $\begin{array}{c}\text { Acc } \\
\%\end{array}$ & 98.3 & 98.1 & 98.2 & 98.7 & 98.4 & 87 \\
\hline
\end{tabular}

TABLE 1: HeARTBEAT RATES USING High-PASS FILTER AND BAND-PASS FILTER WITH AND WITHOUT SMOOTHING.

Accuracy is calculated by the following relation: $100-100 \times \mid \frac{\text { filtered } \text { S21 heartbeat frequency - ECG heartbeat frequency }}{\text { ECG heartbeat frequency }}$ As conclusion, the result obtained from the smoothed highpass filtered phase of $S_{21}$ is more accurate than the result obtained from non-smoothed high pass filtered phase of $S_{21}$, especially because smoothing eliminates noise. Smoothing method is not necessary for band-pass filtered phase of $S_{21}$ because noise is already eliminated by the second cut off frequency. Smoothed high-pass filtered phase of $\mathrm{S}_{21}$ and bandpass filtered phase of $S_{21}$ give accurate results.

\section{CONCLUSION}

Recently, heartbeat rate detection has attracted interest of researchers, especially when traditional electrocardiogram (ECG) measurements with fixed electrodes cannot be achieved. The system used in this work generates a continuous wave toward a person's chest, and detect the reflected one. Using vector network analyzer, the phase variation of $S_{21}$ that carries respiration and heartbeat signals is computed. The vector network analyzer was used to determine optimal frequency with minimum power before implementation process. Filtering method is used to separate heartbeat signal from the phase of $S_{21}$, then peak detection is used to extract the heartbeat rate. The signal processing methods used in this study were applied to real measurements and the obtained results were compared to those given by the ECG taken as reference. Future applications intend to study the heartbeat rate variability HRV obtained from the extracted heartbeat signal and validate the method on measured signals from the person on test.

\section{REFERENCES}

[1] D. Obeid, Touchless cardiopulmonary monitoring: measurements, processing, and modeling. Doctoral Thesis, INSA of Rennes, Sept. 2010, $162 \mathrm{p}$.

[2] M. T. Lynette, J. M. Kudargi, "A review on RF based intruder detection using CW Doppler radar," IOSR Journal of Electronics and Communication Engineering, 2013, pp. 28-31.

[3] B. Lohman, O. B. Lubecke, V. M. Lubecke, P. W. Ong, M. M. Sondhi, "A Digital Signal Processor For Doppler Radar Sensing of Vital Signs,", Engineering in Medicine and Biology Magazine, IEEE, Vol. 21, No.5, 2002, pp.161-164.

[4] D. Obeid, S. Sadek, G. Zaharia, G. El Zein, "A tunable-frequency system for touch-less heartbeat detection and HRV extraction," Proc. of IEEE ISSCS, Iasi, Romania, 2009, 4p.

[5] M. A. Othman, H. Azman, M. N. Husain, M. M. Ismail, H. A. Sulaiman, M. H. Misran, M. A. M. Said, M. Abdulaziz, N. Hassan, A. N. Che Pee, S. Suhaimi, M. R. Motsidi, "Heart Monitoring Systems at $10 \mathrm{GHz}$ Using Microwave Doppler Techniques for Atheletes Fitness Monitoring System: A Review," Australian Journal of Basic and Applied Sciences, Vol. 7, No. 14, Dec., 2013, pp. 57-70.

[6] S. Sadek, L. Ghattas, L. Fawaz, "Wireless microwave sensor for remote monitoring of heart and respiration activity," IEEE Mediterranean Microwave Symposium, Guzelyurt, North Cyprus, 2010, pp. 374-376.

[7] O. B. Lubecke, P. W. Ong, V. M. Lubecke. "10 GHz Doppler Radar Sensing of Respiration and Heart Movement," IEEE Bioengineering Conference, Philadelphia, April 2002, pp. 55-56.

[8] D. Obeid, G. Issa, S. Sadek, G. Zaharia, G. El Zein, "Low Power Microwave Systems for Heartbeat Rate Detection at 2.4, 5.8, 10 and 16 GHz," Proc. of the First International Symp. on Applied Sciences in Biomedical and Comm. Technol. (ISABEL 2008), Aalborg, Denmark, Oct. 2008.

[9] D. Obeid, S. Sadek, G. Zaharia, G. El Zein, "Microwave Doppler Radar for Heart Beat Detection Versus Electrocardiogram: A Validation Approach," MMS 2013, Saida, Lebanon, Oct., 2013. 